\title{
High-degree non-radial modes in the $\delta$ Scuti star AV Ceti
}

\author{
L. Mantegazza and E. Poretti \\ INAF - Osservatorio Astronomico di Brera, Via E. Bianchi 46, 23807 Merate, Italy
}

\begin{abstract}
High-resolution spectroscopic observations taken during 5 consecutive nights of the fast rotating $\delta$ Scuti star AV Ceti $(v \sin i=188 \mathrm{~km} / \mathrm{s})$ show the presence of non-radial modes with $\ell \sim 10-16$ and very short periods $(\nu \sim 40-70 \mathrm{c} / \mathrm{d})$
\end{abstract}

\section{Introduction and Observations}

The rapidly rotating $\delta$ Scuti star AV Ceti has been recently the target of a simultaneous photometric and low-resolution spectroscopic international campaign (Dall et al. 2003 ${ }^{1}$ ). Seven low-degree modes were detected in the frequency range $10-31 \mathrm{c} / \mathrm{d}$.

We observed this star with the Coude Echelle Spectrograph attached at the ESO CAT Telescope during 5 consecutive nights (October 24-30, 1994; Remote Control from Garching headquarters) for about 2.5 consecutive hours per night and 55 spectrograms were collected with exposure times of $10 \mathrm{~min}$ and a typical $\mathrm{S} / \mathrm{N}$ ratio at the continuum level of about 230 . They had a resolution of 60000 and cover the region 4494-4513 $\AA$. In this region we have two useful lines to study line profile variations: Till $4501 \AA$ and Fell $4508 \AA \AA$. Fig. 1 shows the average continuum normalized spectrum (upper panel) and the pixel-by-pixel standard deviation (lower panel).

\footnotetext{
${ }^{1}$ Added by editor (MB): The Astronomy and Astrophysics publisher used an incorrect name for the star (AV Cetei instead of AV Ceti) in the title and running head of the Dall et al. paper. The authors of the present publication note: Cetus, $-i$ belongs to the second latin declination (as, for example, Cepheus,-i) : nominative and genitive cases are different (Cephei, Ceti). On the other hand, Doradus,-us belongs to the fourth declination and the genitive is the same as the nominative $(\gamma$ Doradus, not $\gamma$ Doradi $)$. For a correct use of abbreviations of constellations see http://www.iau.org/Activities/nomenclature/const.html
} 


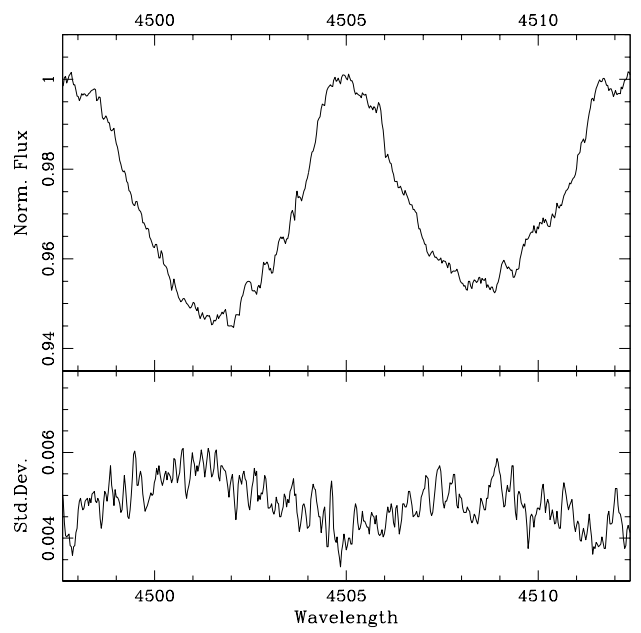

Figure 1: Average normalized spectrum (top panel); pixel-by-pixel data standard deviation (lower panel).

\section{Data analysis and discussion}

We can see that the line profiles have variations with a std.dev of about 0.002 of the continuum level. A non-linear least-squares fit of a rotationally broadened Gaussian intrinsic profile, taking also into account the limb darkening, on the two average line profiles supplies $v \sin i=188 \pm 1 \mathrm{~km} / \mathrm{s}$.

The line profile variations were analyzed by means of the Fourier Doppler Imaging Technique (Kennelly et al. 1998; Hao 1998). Fig. 2 shows the twodimensional power spectrum obtained considering all the data in a single time series and merging the information of the two spectral lines. We see that there is a clear clump of modes in the region $10 \leq \ell \leq 16,35 \leq \nu \leq 70 \mathrm{c} / \mathrm{d}$. Because of the very severe aliasing, due to the poor temporal coverage, it is not possible to derive a detection of individual modes.

A CLEANed version of this power spectrum is shown in Fig. 3 (gain=0.9, 100 iterations). This figure should be considered only as indicative, and the individual modes cannot be taken at face value, since the poor spectral window makes a correct deconvolution problematic. However it clearly shows that we should expect a very complex pulsation pattern with several high-degree, high-frequency modes. Can this be due to the fast rotation? More intensive 


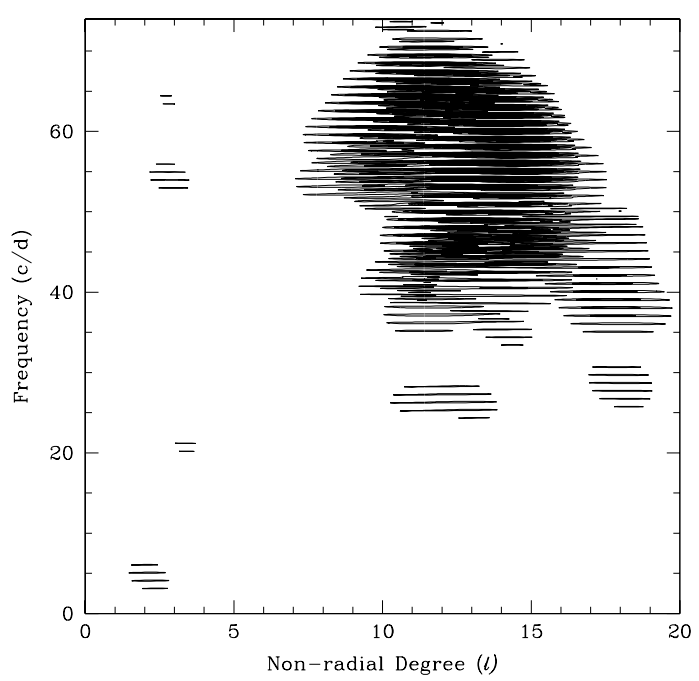

Figure 2: Two-dimensional Fourier power spectrum.

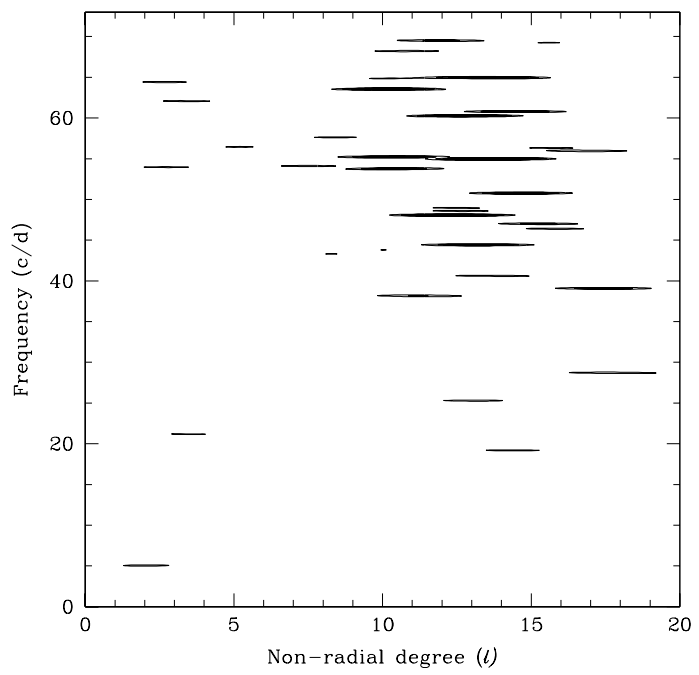

Figure 3: CLEANed two-dimensional Fourier power spectrum. 
observations are necessary to get a more exhaustive picture of the stellar pulsation spectrum. We also note that the low-degree modes, photometrically detected by Dall et al. (2003) do not produce relevant patterns in our power spectrum. This does not necessarily mean that they are not present, since our technique is more sensitive to high-degree modes (see also Mantegazza 2000).

\section{References}

Dall, T.H., Handler, G., Moalusi, M.B., Frandsen, S. 2003, A\&A 410, 983

Hao, J. 1998, ApJ 500, 440

Kennelly,E.J. et al. 1998, ApJ 495, 440

Mantegazza, L. 2000, ASP Conf. Ser. 210, 138 